\title{
Circular RNA Circ_0016760 Modulates Non-Small-Cell Lung Cancer Growth Through the miR-577/ZBTB7A Axis
}

This article was published in the following Dove Press journal: Cancer Management and Research

\section{Yanhong $\mathrm{Hao}^{\mathrm{l}, *}$ Junfeng $\mathrm{Xi}^{1, *}$ Yancai Peng' \\ Burong Bian ${ }^{2}$ Guangjun $\mathrm{HaO}^{2}$ Yunfeng $\mathrm{Xi}^{3}$ Zhibin Zhang'}

'Department of Cardiothoracic Surgery, The First Hospital of Yulin, Yulin City, Shanxi Province, People's Republic of China; ${ }^{2}$ Department of Oncology, The First Hospital of Yulin, Yulin City, Shanxi Province, People's Republic of China; ${ }^{3}$ Department of Dermatological Surgery, The First Hospital of Yulin, Yulin City, Shanxi Province, People's Republic of China

*These authors contributed equally to this work
Correspondence: Zhibin Zhang Email r7mj90@I63.com
Background: Patients with locally advanced or metastatic non-small-cell lung cancer (NSCLC) have a poor prognosis. Circular RNA circ_0016760 (circ_0016760) is associated with the development of NSCLC. At present, the role and regulatory mechanism of circ_0016760 in NSCLC have not been well explained.

Methods: Quantitative real-time polymerase chain reaction (qRT-PCR) was executed to detect the expression of circ 0016760, miR-577, and Zinc finger and BTB domain containing 7A (ZBTB7A) mRNA in NSCLC tissues and cells. The colony formation, migration, invasion, and extracellular acidification rate (ECAR) of NSCLC cells were determined through colony formation, transwell, or ECAR assays. The relationship between circ_0016760 or ZBTB7A and miR-577 was analyzed via dual-luciferase reporter and RNA pull-down or RNA immunoprecipitation (RIP) assays. Protein level of ZBTB7A was evaluated with Western blot analysis. Xenograft assay was conducted to confirm the role of circ 0016760 in vivo.

Results: Circ_0016760 and ZBTB7A were upregulated and miR-577 was downregulated in NSCLC tissues and cells. Circ_0016760 exhaustion curbed the colony formation, migration, invasion, and ECAR of NSCLC cells in vitro and impeded tumor growth in vivo. Mechanically, circ_0016760 modulated ZBTB7A expression via sponging miR-577 in NSCLC cells. MiR-577 downregulation abolished the repressive effects of circ 0016760 silencing on colony formation, migration, invasion, and ECAR of NSCLC cells. Also, ZBTB7A upregulation overturned the repressive impacts of miR-577 elevation on colony formation, migration, invasion, and ECAR of NSCLC cells.

Conclusion: Circ_0016760 silencing impeded NSCLC advancement through regulation of the miR-577/ZBTB7A axis.

Keywords: circ_0016760, miR-577, ZBTB7A, NSCLC, ECAR

\section{Introduction}

Although much progress has been made in diagnosis and treatment, lung cancer remains the leading cause of cancer morbidity and mortality in the world. ${ }^{1}$ It was reported that lung cancer deaths in 2018 were close to one-fifth of cancer deaths. ${ }^{2}$ Also, non-small-cell lung cancer (NSCLC) accounts for more than $85 \%$ of all lung cancers. ${ }^{3}$ Besides, most NSCLC patients present with locally advanced or metastatic disease at the time of diagnosis and have a poor prognosis. ${ }^{4}$ Hence, it is indispensable to study the advancement of NSCLC for NSCLC treatment. 
Circular RNAs (circRNAs) are a type of non-coding RNAs with a circular covalent-closed structure. ${ }^{5}$ Studies have revealed that circRNAs can act as a sponge for microRNAs (miRNAs), participate in the formation of RNA-protein complexes, and modulate splicing and transcription of targeted genes. ${ }^{6,7}$ Moreover, circRNAs were pointed out to be connected with the tumorgenesis of a range of cancers $^{8-10}$ For instance, CircRNA circAGFG1 contributed to the development of triplenegative breast cancer. ${ }^{10}$ Also, circRNA_102171 accelerated papillary thyroid cancer development. ${ }^{11}$ Circular RNA circ_0016760 (circ_0016760) is generated from the chromosome region 1q42.13 and its associated-gene symbol is synaptosome associated protein 47. Previous research claimed that circ_0016760 promoted the development of NSCLC. ${ }^{12}$ However, there are few studies on the pathogenesis of circ_0016760 in NSCLC.

MiRNAs are another outstanding non-coding RNAs that modulate gene expression through silencing specific target messenger RNA at the post-transcriptional level. ${ }^{13}$ They exert a carcinogenic or suppressive function in many cancers by altering the expression of oncogenes or tumor suppressor genes. ${ }^{14}$ MicroRNA-577 (miR-577) has been disclosed to be related to the development of multiple cancers. ${ }^{15-18}$ MiR-577 repressed cell proliferation, invasion, and migration in papillary thyroid cancer cells via downregulating SPHK2. ${ }^{16}$ Also, miR-577 facilitated tumor chemoresistance and metastasis in gastric cancer. ${ }^{15}$ Moreover, miR-577 was implicated in the progression of NSCLC. ${ }^{19,20}$ Nevertheless, it is unclear whether miR-577 is regulated by circ_0016760 in the NSCLC cells.

Zinc finger and BTB domain-containing 7A (ZBTB7A), also known as TIP21, pokemon, or FBI1, is a member of the POK family of transcriptional suppressors. Studies have indicated that ZBTB7A is involved in the development of a variety of cancers, such as melanoma, prostate cancer, breast cancer, and osteosarcoma. ${ }^{21-24}$ Furthermore, silenced ZBTB7A expression repressed the development of NSCLC. ${ }^{25}$ At present, it is indistinct whether ZBTB7A participates in the NSCLC progression regulated by circ_0016760.

Hence, we explored the expression pattern of circ_0016760 in NSCLC tissues and cells. Furthermore, the role of circ_0016760 in NSCLC cells was investigated. Additionally, the molecular mechanism of the circ_0016760/miR-577/ZBTB7A axis in NSCLC was studied. This study provided a theoretical basis for the treatment of NSCLC.

\section{Materials and Methods NSCLC Specimen Collection}

The research was sanctified by the Ethics Committee of the First Hospital of Yulin. 47 paired NSCLC tissues and neighboring normal NSCLC tissues were converged from NSCLC patients who underwent surgical resection at the First Hospital of Yulin. All NSCLC patients who participated in the study did not receive radiotherapy or chemotherapy before surgery. Written informed consents were obtained from all participants. The research was performed in accordance with the Declaration Helsinki principles.

\section{Cell Culture and Transfection}

NSCLC cell lines (A549, H1299, and H1975) were procured from American Tissue Culture Collection (Manassas, VA, USA). Human bronchial epithelial cells 16HBE were obtained from Bena Culture Collection (Suzhou, Jiangsu). The Dulbecco's Modified Eagle Medium (DMEM; Gibco, Grand Island, NY, USA) was employed to maintain the above cell lines. All cells were kept in an incubator with 5\% $\mathrm{CO}_{2}$ at $37^{\circ} \mathrm{C}$. Additionally, fetal bovine serum (10\%, FBS, Gibco) and streptomycin/penicillin (1\%, HyClone, Logan, UT, USA) were added to DMEM in order to maintain cell growth.

Small interference RNA targeting circ_0016760 (sicirc_0016760) and negative control (si-NC) were procured from GenePharma (Shanghai, China). MiR-577 mimics and inhibitors (miR-577 and anti-miR-577) and their negative controls (miR-NC and anti-NC) were obtained from Ambion Inc (Austin, TX, USA). The ZBTB7A overexpression vectors (ZBTB7A) were constructed using the pcDNA3.1 vector (vector; Invitrogen, Carlsbad, CA, USA). Lipofectamine 3000 reagent (Invitrogen) was utilized to transfect oligonucleotides or vectors into A549 and $\mathrm{H} 1299$ cells.

\section{Quantitative Real-Time Polymerase Chain Reaction (qRT-PCR)}

The TRIzol Reagent from Thermo Fisher Scientific (Waltham, MA, USA) was applied to extract total RNA from NSCLC specimens and cells. The NE-PER Nuclear and Cytoplasmic Extraction Reagents (Thermo Fisher Scientific) was used to isolate the nuclear and cytoplasmic fractions of the A549 and H1299 cells. High-Capacity complementary DNA Reverse Transcription kit (Thermo Fisher Scientific) or MiRNA Reverse Transcription kit (Thermo Fisher Scientific) was utilized to synthesize the 
first-strand complementary DNA for circ_0016760, ZBTB7A, and miR-577, respectively. RNase R digestion was performed at a ratio of $3 \mathrm{U}$ enzyme $/ 1 \mathrm{mg}$ RNA. ${ }^{26}$ QRTPCR was assessed using the SYBR green PCR master mixes (Thermo Fisher Scientific). The primers were exhibited as follows: glyceraldehyde-3-phosphate dehydrogenase (GAPDH) (F: 5'-GAAGGTGAAGGTCGGAGTC-3', R: 5'GAAGATGGTGATGGGATTTC-3'), circ_0016760 (F: 5'GCTGTCGCTCAGGATCGTTT-3', R: 5'-TTGCGCTTCT GAGCACCAAT-3'), ZBTB7A (F: 5'-GCTTGGGCCGGTT GAATGTA-3', R: 5'-GGCTGTGAAGTTACCGTCGG-3'), 18S rRNA (F: 5'-GTCAGAGGTGAAATTCTTGGATTTA -3', R: 5'-AGGGCAGGGACGTAATCAACG-3'), U6 small nuclear RNA (snRNA) (F: 5'-GCTCGCTTCGGCAGC ACA-3'，R: 5'-GAGGTATTCGCACCAGAGGA-3'), and miR-577 (F: 5'-TGCGGTAGATAAAATATTGG-3', R: 5'CCAGTGCAGGGTCCGAGGT- $3^{\prime}$ ). The levels of circ_0016760, ZBTB7A, and miR-577 were computed through $2^{-\Delta \Delta \mathrm{Ct}}$ method, and GAPDH or U6 snRNA was served as an internal control.

\section{Colony Formation Assay}

In short, the transfected A549 and H1299 cells $\left(1 \times 10^{2}\right.$ cells) were seeded in the cell culture dish, and the medium was changed every 3 days. After culture for 9 days, the cells were fixed with ethanol (75\%) and stained with crystal violet $(0.2 \%$, KeyGen, Jiangsu, China) for $2 \mathrm{~h}$, respectively. Colonies (> 50 cells/colony) were counted and photographed using the light microscope (Olympus, Tokyo, Japan). ${ }^{27}$

\section{Transwell Assay}

For the migration assay, DMEM with FBS (10\%) was supplemented into the lower of the transwell chamber ( 8 $\mu \mathrm{m}, \quad \mathrm{BD}$ Biosciences, San Jose, CA, USA) as a chemoattractant. Contemporaneously, DMEM $(200 \mu \mathrm{L})$ containing the cells $\left(5 \times 10^{4}\right)$ was added to the top of the transwell chamber. After culturing for $24 \mathrm{~h}$, the cells on the upper of the membrane were scraped with a cotton swab. Then, the cells on the lower surface of the membrane were fixed and stained with methanol $(100 \%)$ and crystal violet $(0.25 \%$, KeyGen), respectively. For the invasion assay, DMEM containing FBS $(10 \%)$ was added to the lower chamber of the transwell chamber coated with matrigel matrix (BD Biosciences). Then, DMEM $(200 \mu \mathrm{L})$ embracing the cells $\left(5 \times 10^{4}\right)$ was placed into the upper chamber of the transwell chamber. The cells on the lower surface of the membrane were fixed with methanol
$(100 \%)$ and stained with crystal violet $(0.25 \%$, KeyGen) after culture for $24 \mathrm{~h}$. The number of migrating or invading cells was counted using a light microscope (Olympus).

\section{Extracellular Acidification Rate (ECAR) Measurement Assay}

According to previous studies, the glycolysis was analyzed via monitoring the ECAR by the Seahorse $\mathrm{XF}^{\mathrm{e}} 96$ Extracellular Flux Analyzer (Seahorse Bioscience, North Billerica, MA, USA). ${ }^{28}$ In brief, the transfected A549 and H1299 cells (approximately $2 \times 10^{4}$ ) were seeded into the cell culture microplate matched for the Seahorse $\mathrm{XF}^{\mathrm{e}} 96$. Glucose, the oxidative phosphorylation inhibitor oligomycin, and the glycolysis inhibitor 2-deoxy-glucose were sequentially injected into each well at the agreed time point after baseline measurement. The data were analyzed through the Seahorse $\mathrm{XF}^{\mathrm{e}} 96$ Wave software and displayed in $\mathrm{mPH} / \mathrm{min}$.

\section{Dual-Luciferase Reporter Assay}

The starBase 3.0 was employed for the prediction of the binding sites between circ_0016760 or ZBTB7A and miR577. Afterward, the sequence of wild type (WT) circ_0016760 or the $3^{\prime}$ Untranslated Region (UTR) fragment of ZBTB7A harboring miR-577 binding sites was amplified and inserted into pGL3-control vector (Promega) for the construction of the luciferase reporters. Also, the sequences of mutant (MUT) circ_0016760 sequence and the $3^{\prime} \mathrm{UTR}$ of ZBTB7A were inserted into the pGL3control vector to construct the luciferase reporters. The luciferase reporters and miR-NC or miR-577 were cotransfected into A549 and H1299 cells using Lipofectamine 3000 reagent. After transfection for $48 \mathrm{~h}$, the luciferase activities were evaluated via the dual-luciferase reporter assay kit (Promega).

\section{RNA Pull-Down Assay}

A549 and H1299 cells were transfected with biotinylated (bio)-miR-NC or bio-miR-577. After washing with PBS, the cells were lysed with specific lysis buffer (Amnion, Austin, Texas, USA). Thereafter, the lysate was incubated with M-280 streptavidin magnetic beads (Sigma, Louis, Missouri, MO, USA) for $3 \mathrm{~h}$. Next, the bound RNA was exacted with Trizol reagent. The enrichment level of circ_0016760 was assessed with qRT-PCR. 


\section{RNA Immunoprecipitation (RIP) Assay}

The Magna RIP kit (Millipore, Bedford, MA, USA) was employed to conduct the RIP assay. In short, A549 and H1299 cells were lysed with the RIP lysis buffer. Thereafter, the lysates were incubated in the RIP buffer embracing magnetic beads conjugated with anti-IgG or anti-Ago2 antibodies (Millipore) for $4 \mathrm{~h}$. After that, the RNA was isolated from the magnetic beads using the TRIzol reagent. The abundance of miR-577 and ZBTB7A was analyzed by qRT-PCR.

\section{Western Blot Analysis}

The radio-immunoprecipitation assay (RIPA) lysis buffer (Thermo Fisher Scientific) was used to extract total protein from NSCLC specimens and cells. The extracted total protein was separated through the sodium dodecyl sulphate-polyacrylamide gel electrophoresis (SDS-PAGE, $10 \%$ ). Next, the separated proteins were transferred onto polyvinylidene difluoride (PVDF; Millipore, Bedford, MA, USA) membranes via the wet electrophoretic transfer method. Afterward, the PVDF membranes were immersed in Tris Buffered Saline Tween (TBST) buffer with 5\% skim milk for $1 \mathrm{~h}$. After washing with PBS, the PVDF membranes were incubated with rabbit anti-ZBTB7A (1:10,000, ab175918, Abcam, Cambridge, MA, USA) or rabbit anti- $\beta$-actin (1:200, ab115777, Abcam). $\beta$-actin was used as a loading control. Then, the membranes were incubated with the secondary antibody goat anti-rabbit $\operatorname{IgG}$ (1:2000, ab205718, Abcam). The Immobilon TM Western Chemiluminescent HRP Substrate (Millipore) was utilized to visualize the protein bands. The Bio-Rad Quantity One (Bio-Rad, Hercules, CA, USA) was applied to quantify the protein bands.

\section{Xenograft Assay}

The animal experiment was ratified by the Animal Ethics Committee of the First Hospital of Yulin, which was in accordance with Institutional Animal Care and Use Committee and Animal studies were performed in compliance with the ARRIVE guidelines and the Basel Declaration. Mouse xenograft models were established using $10 \mathrm{BALB} / \mathrm{c}$ nude mice (4-6 weeks old) from the Shanghai Experimental Animal Center (Shanghai, China). Briefly, lentivirus-mediated short hairpin RNA targeting circ_0016760 (sh-circ_0016760) or its negative control (sh-NC) was transfected into A549 cells. Then, A549 cells with sh-circ_0016760 or sh-NC were subcutaneously injected into the dorsal side of nude mice (5 mice/group). The tumor volume was measured every 7 days with a digital caliper and calculated with the equation: Volume $=\left(\right.$ length $\times$ width $\left.^{2}\right) / 2$. After injection 28 days, the mice were euthanized for subsequent studies.

\section{Statistical Analysis}

The experiments in this study in vitro were executed at least 3 replicates. SPSS 19.0 software (SPSS, Chicago, IL, USA) and GraphPad Prism 6.0 (GraphPad, San Diego, CA, USA) were executed for statistical analysis. The data were shown as mean \pm standard deviation. The differences between the two groups were analyzed through the Student's $t$ test. One-way variance analysis (ANOVA) was employed to compare the differences between three or more groups. The correlation between miR-577 and circ_0016760 or ZBTB7A expression as determined with Pearson's correlation analysis. $P<0.05$ was deemed statistically significant.

\section{Results \\ Circ_0016760 Was Upregulated in NSCLC Tissues and Cells}

At the outset, we detected circ_0016760 expression in 47 paired NSCLC tissues and neighboring normal tissues through qRT-PCR. Compared to the neighboring normal tissues, circ_0016760 was elevated in NSCLC tissues (Figure 1A). Likewise, we observed that circ_0016760 expression was prominently increased in NSCLC cells (A549, H1299, and H1975) than that in the 16HBE cells, and circ_0016760 levels were apparently higher in A549 and H1299 cells (Figure 1B). Subsequently, we assessed the expression of circ_0016760 and GAPDH in A549 and H1299 cells with or without RNase R treatment with qRTPCR. The results presented that there was no prominent difference in circ_0016760 expression in A549 and H1299 cells with or without RNase R treatment. However, the levels of linear GAPDH were reduced in A549 and H1299 cells after RNase R treatment compared with the control group (Figure 1C and D). Additionally, we assessed the levels of circ_0016760 in the cytoplasm and nuclear fractions of A549 and H1299 cells via qRT-PCR. As exhibited in Figure 1E and F, circ_0016760 levels were strikingly enriched in the cytoplasm fraction of A549 and H1299 cells, implying that circ_0016760 was principally localized in the cytoplasm of the cells. Collectively, these 

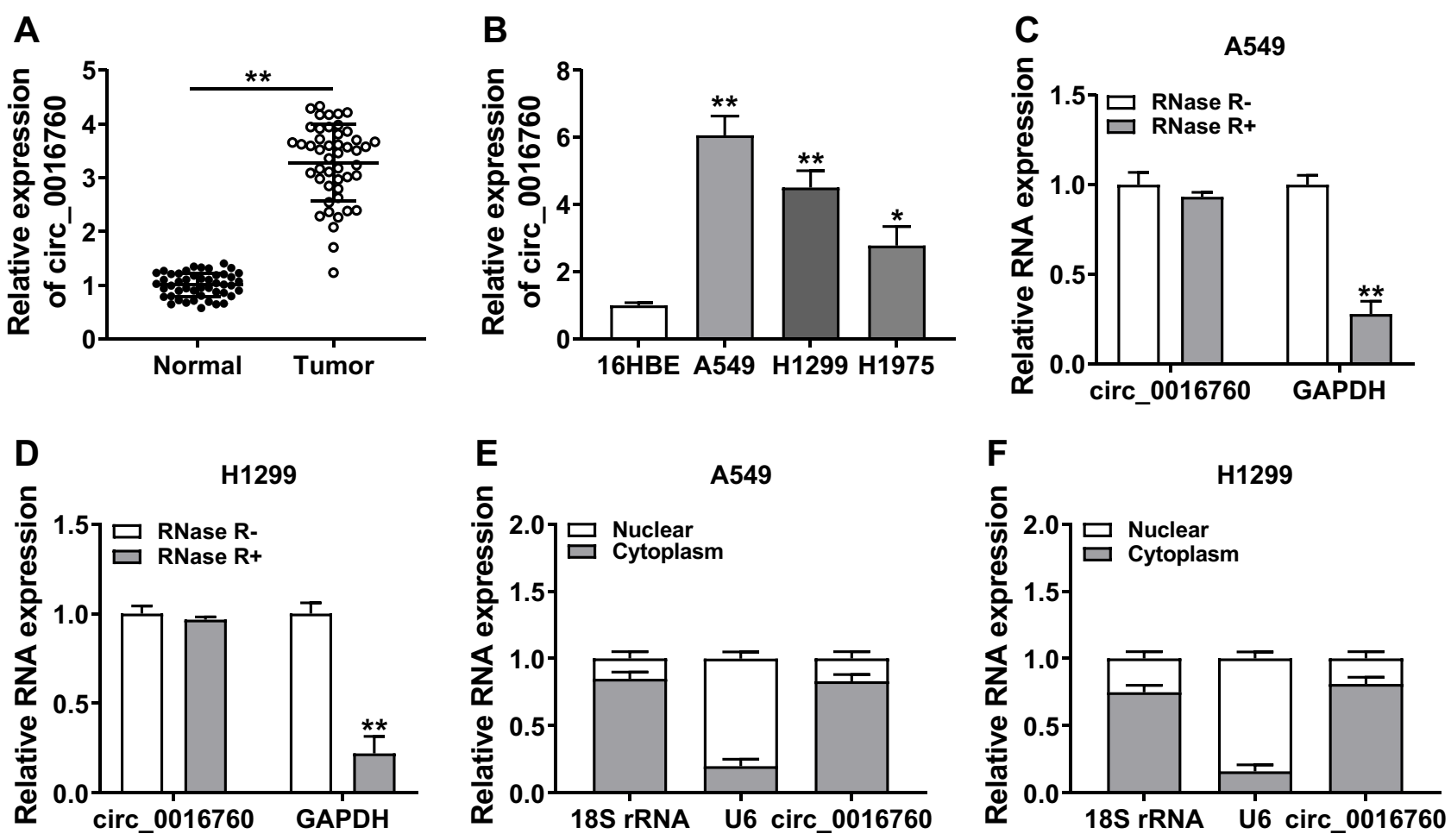

Figure I Expression and characterization of circ_0016760 in NSCLC. (A and B) The expression of circ_0016760 in NSCLC tissues and neighboring normal tissues, as well as NSCLC cells (A549, HI299, and HI975) and the I6HBE cells, was analyzed through qRT-PCR. (C and D) Effect of RNase R on the level of circ_0016760 and GAPDH of A549 and HI299 cells was analyzed through qRT-PCR. (E and F) The distribution of circ_0016760 in A549 and HI 299 cells was determined with qRT-PCR. *P < 0.05 and $* * P<0.01$.

findings suggested that circ_0016760 might exert a cancerogenic role in NSCLC.

\section{Depletion of Circ_0016760 Curbed Colony Formation, Migration, Invasion, and Decreased ECAR of NSCLC Cells}

To explore the role of circ_0016760 in NSCLC, we first assessed the expression of circ_0016760 in A549 and H1299 cells transfected with si-circ_0016760 or si-NC. The results displayed that circ_0016760 was dramatically downregulated in A549 and H1299 cells transfected with sicirc 0016760 when compared to the si-NC group, indicating that the si-circ_0016760 could be used for subsequent studies (Figure 2A). Results of colony formation assay demonstrated that decreased circ_0016760 expression markedly repressed the colony formation ability of A549 and H1299 cells (Figure 2B). Moreover, transwell assay presented that the migration and invasion of circ_0016760silenced A549 and H1299 cells were remarkably impeded relative to the control group (Figure $2 \mathrm{C}$ and $\mathrm{D}$ ). Also, ECAR assay showed that the ECAR was obviously declined in A549 and H1299 cells after si-circ_0016760 transfection (Figure 2E). These data demonstrated that circ_0016760 knockdown suppressed cell colony formation, migration, invasion, and reduced ECAR in NSCLC cells.

\section{MiR-577 Served as a Target for Circ 0016760}

To probe into the molecular mechanism of circ_0016760 in NSCLC, we employed starBase 3.0 to predict the possible targets for circ_0016760. As displayed in Figure 3A, miR577 possessed the potential binding sites for circ_0016760. Dual-luciferase reporter assay manifested that enhanced miR-577 expression evidently repressed the luciferase activity of the luciferase reporters containing circ_0016760-WT in comparison with the miR-NC group, but there was no marked change in the luciferase reporters embracing circ_0016760-MUT (Figure 3B and C). RNA pull-down assay manifested that the level of circ_0016760 was overtly enriched in the bio-miR-577 group compared to the bio-miR$\mathrm{NC}$ group (Figure 3D). We discovered that miR-577 was specially downregulated in NSCLC tissues in contrast to the neighboring normal tissues (Figure 3E). Pearson's 

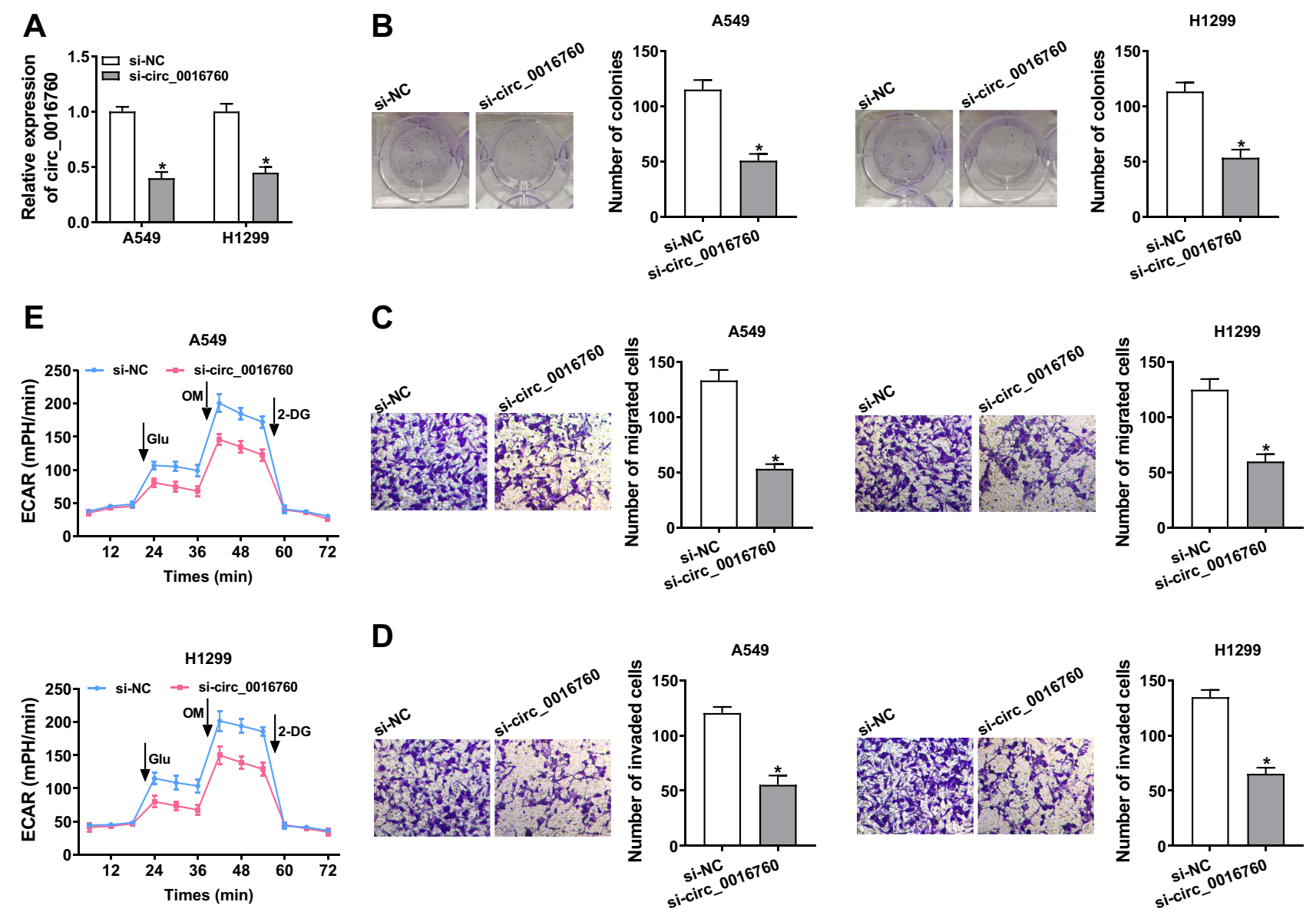

Figure 2 Impacts of circ_0016760 silencing on colony formation, migration, invasion, and ECAR of NSCLC cells. (A-E) A549 and HI299 cells were transfected with si-circ _0016760 or si-NC. (A) QRT-PCR was performed to assess the expression of circ_0016760 in A549 and HI299 cells. (B) Colony formation assay was carried out to determine the colony formation ability of A549 and HI299 cells. (C and D) Transwell assay was executed for the assessment of the migration and invasion capacities of A549 and HI299 cells. (E) The ECAR of A549 and HI299 cells was analyzed through ECAR assay. *P $<0.05$.

correlation analysis showed that the expression of miR-577 was negatively correlated with circ_0016760 in NSCLC tissues (Figure 3F). MiR-577 was also markedly decreased in A549 and H1299 cells compared to the 16HBE cells (Figure 3G). MiR-577 expression was enhanced in circ_0016760-inhibited A549 and H1299 cells (Figure 3H). Taken together, these results indicated that circ_0016760 acted as a sponge for miR-577 in NSCLC cells.

\section{MiR-577 Silencing Overturned}

\section{Circ_0016760 Repression-Mediated Influence on Colony Formation,} Migration, Invasion, and ECAR of NSCLC Cells

Knowing that miR-577 was negatively regulated by circ_0016760 in NSCLC cells, we further investigated whether circ_0016760 played its function in NSCLC cells via miR-577. We first evaluated the expression levels of miR-577 in A549 and H1299 cells transfected with antimiR-577 or anti-NC. The results showed that miR-577 expression was obviously reduced in A549 and H1299 cells transfected with anti-miR-577 compared to that in the anti-NC group (Figure 4A). Colony formation assay revealed that miR-577 downregulation reversed the suppression of colony formation of A549 and H1299 cells induced by circ_0016760 inhibition (Figure 4B). Moreover, decreased miR-577 expression restored the repressive effects of circ_0016760 exhaustion on cell migration and invasion in A549 and H1299 cells (Figure 4C and D). Additionally, ECAR assay also exhibited that the reduction of ECAR of A549 and H1299 cells caused by the inhibition of circ_0016760 was recovered by miR577 silencing (Figure 4E). Together, these findings implied 


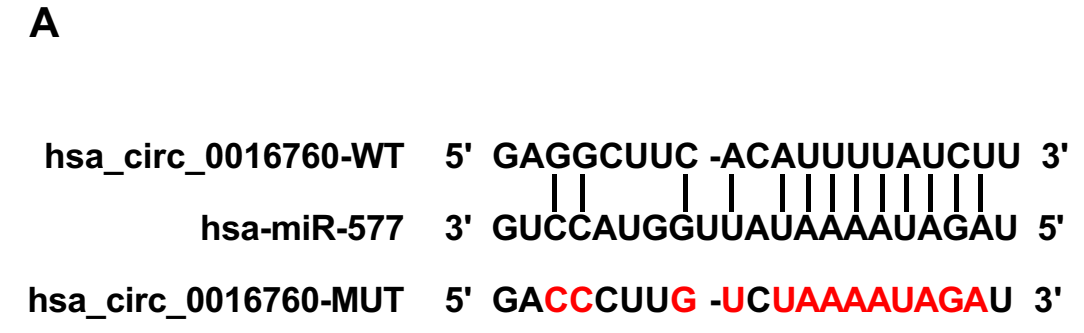

C

H1299
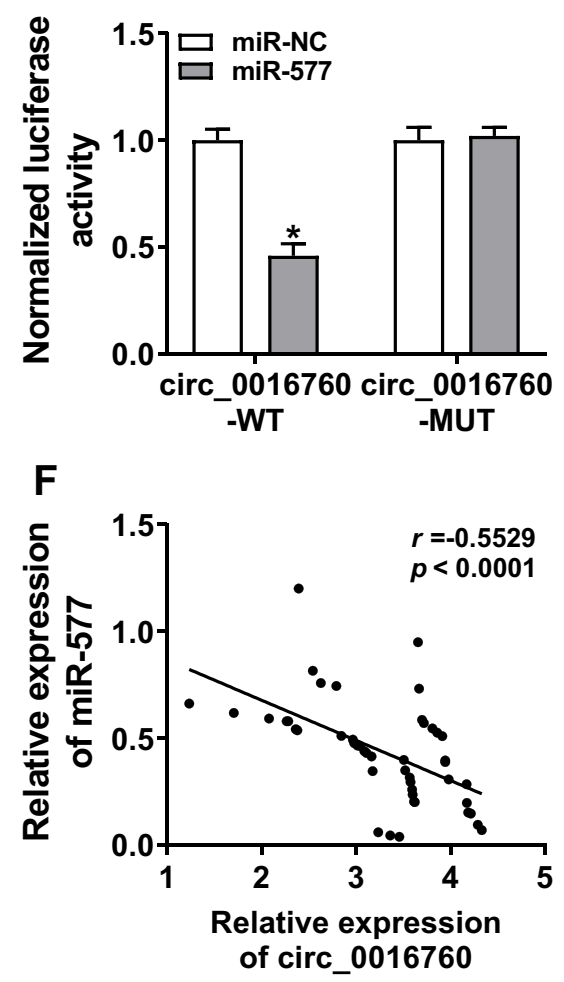

D

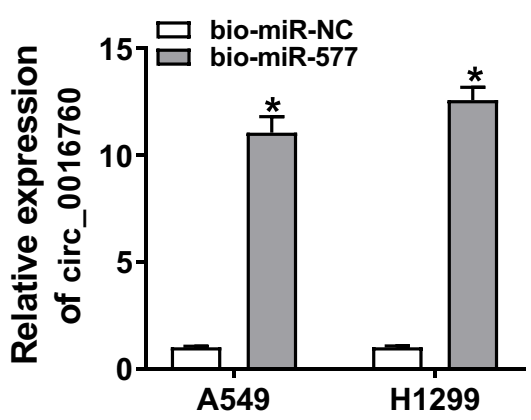

G

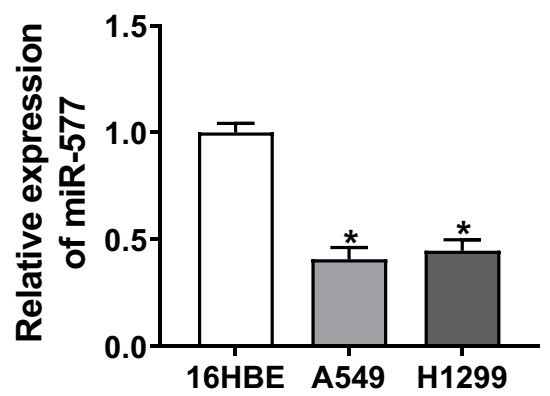

A549

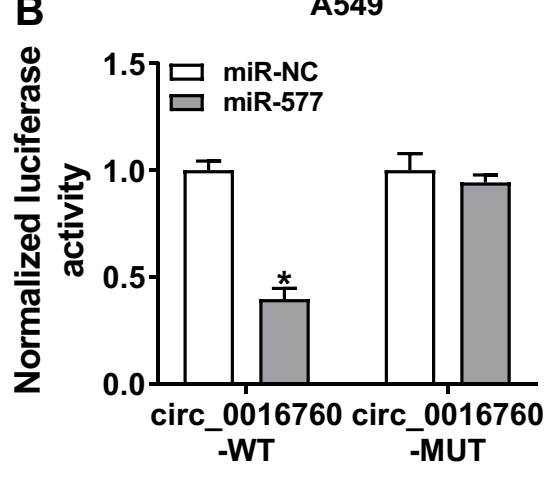

E

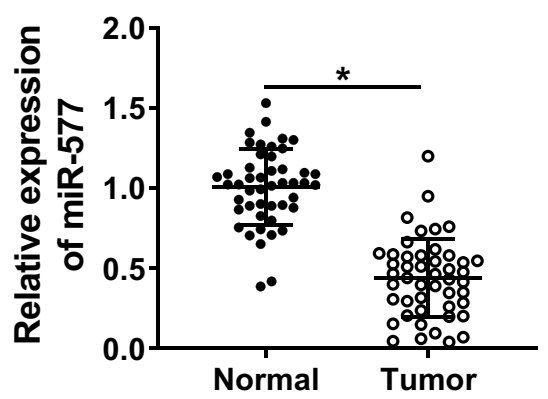

H

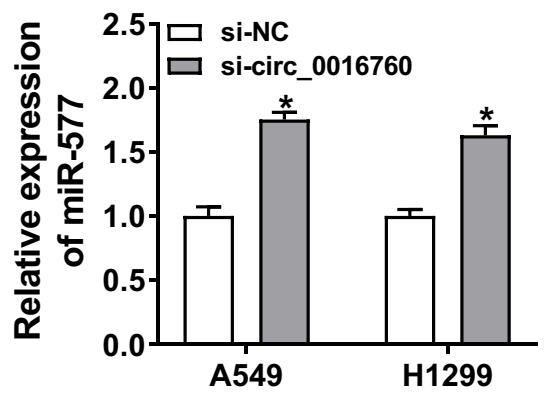

Figure 3 MiR-577 was negatively regulated by circ_0016760 in NSCLC cells. (A) The binding sites of circ_0016760 in miR-577 were predicted with the starBase 3.0 . (B and C) The luciferase activities of circ_0016760-WT and circ_0016760-MUT in A549 and HI299 cells transfected with miR-577 or miR-NC were evaluated via dual-luciferase reporter assay. (D) After RNA pull-down assay, the enrichment of circ_00I6760 was assessed with qRT-PCR. (E) QRT-PCR was utilized to analyze the expression of miR577 in NSCLC tissues and neighboring normal tissues. (F) The correlation between circ_0016760 and miR-577 in NSCLC tissues was analyzed via Pearson's correlation analysis. (G) QRT-PCR was used to assess the expression of miR-577 in A549, HI299, and I6HBE cells. (H) Influence of circ_00।6760 repression on the expression of miR577 of A549 and HI299 cells was explored via qRT-PCR. *P $<0.05$.

that circ_0016760 modulated the colony formation, migration, invasion, and ECAR of NSCLC cells via miR-577.

\section{ZBTB7A Was Identified as a Target for miR-577}

In view of the above results, we further explored the targets for miR-577 through the starBase 3.0. We discovered that ZBTB7A had the possible binding sites for miR-577 (Figure 5A). Dual-luciferase reporter assay manifested that miR-577 overexpression dramatically reduced the luciferase activity of the luciferase reporters with ZBTB7A-WT, while the luciferase activity of the luciferase reporters with ZBTB7A-MUT did not change (Figure 5B and $\mathrm{C}$ ). RIP assay exhibited that miR-577 and ZBTB7A were overtly enriched in Ago2containing ribonucleoprotein complexes, indicating that Ago 2 protein directly bound to miR-577 and ZBTB7A 
A

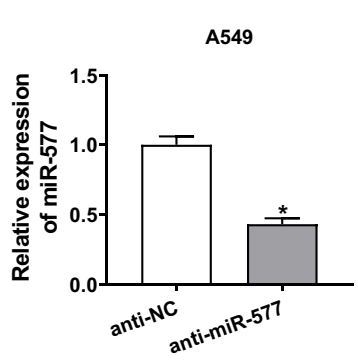

C

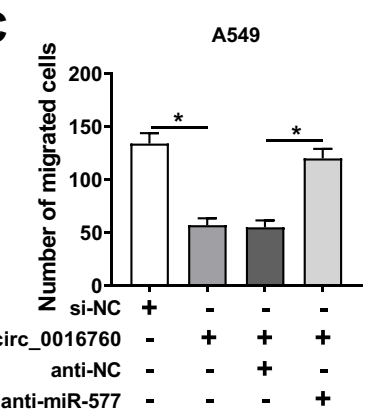

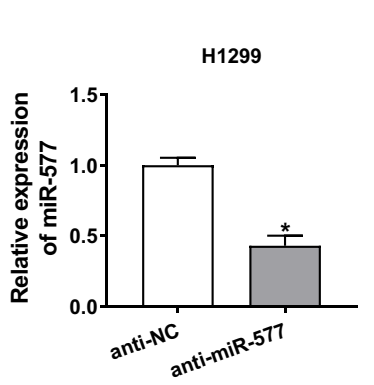

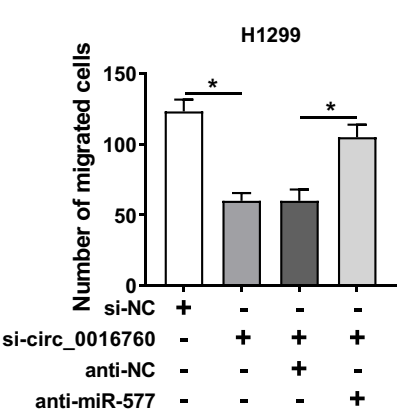

B

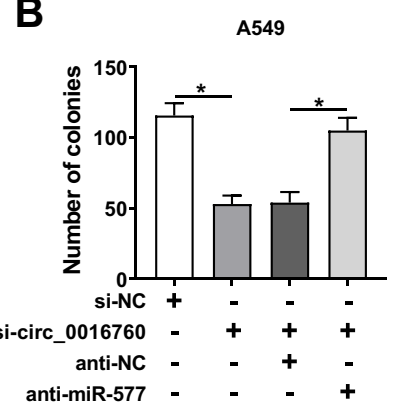

D

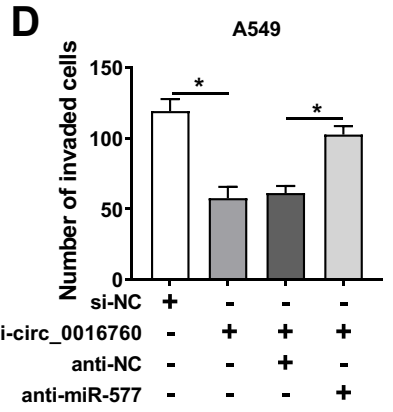

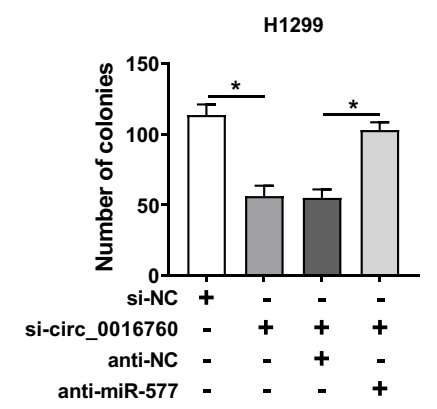

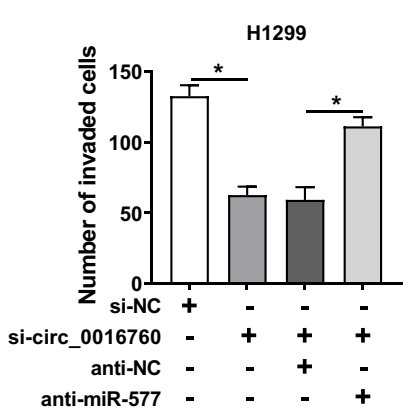

E

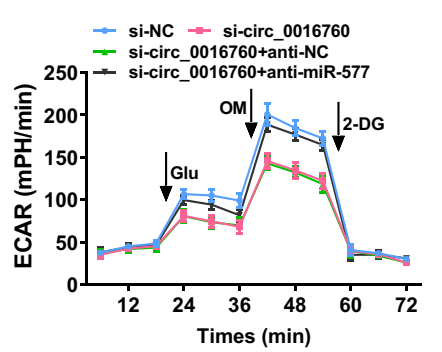

H1299

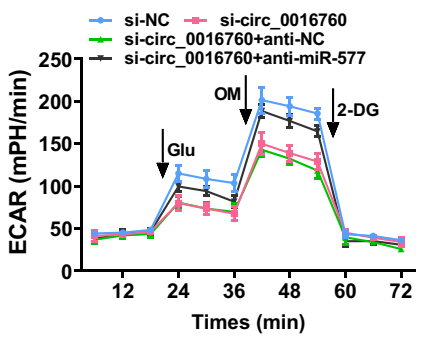

Figure 4 Circ_0016760 exerted its function in NSCLC cells via miR-577. (A) The expression of circ_0016760 in A549 and HI299 cells transfected with anti-miR-577 or anti-NC was detected through qRT-PCR. (B-E) A549 and HI 299 cells were transfected with si-NC, si-circ_0016760, si-circ_0016760+anti-NC, or si-circ_00I6760+antimiR-577. (B) The colony formation capacity of A549 and HI299 cells was determined via colony formation assay. (C and D) The migration and invasion abilities of A549 and HI299 cells were assessed with transwell assay. (E) ECAR assay was employed to analyze the ECAR of A549 and HI299 cells. *P < 0.05.

in A549 and H1299 cells (Figure 5D and E). Also, the levels of ZBTB7A mRNA and protein were evidently upregulated in NSCLC tissues compared with the neighboring normal tissues (Figure $5 \mathrm{~F}$ and $\mathrm{G}$ ). And ZBTB7A mRNA and protein were also enhanced in A549 and H1299 cells than that in the 16HBE cells (Figure 5H and I). Besides, Pearson's correlation analysis showed that ZBTB7A and miR-577 had a negative correlation in NSCLC tissues (Figure 5J). And miR-577 inhibition obviously elevated the levels of ZBTB7A mRNA and protein in A549 and H1299 cells (Figure $5 \mathrm{~K}$ and $\mathrm{L}$ ). These data suggested that ZBTB7A acted as a target of miR-577 in NSCLC cells.

\section{ZBTB7A Elevation Abolished miR-577 Overexpression-Mediated Effects on Colony Formation, Migration, Invasion, and ECAR of NSCLC Cells}

Considering that ZBTB7A served as a target for miR-577, we further explored whether miR-577 mediated the colony formation, migration, invasion, and ECAR of NSCLC cells via ZBTB7A. Western blot analysis exhibited that the protein level of ZBTB7A was notably enhanced in A549 and H1299 cells transfected with ZBTB7A than that in the vector group (Figure 6A). Increased miR-577 expression obviously repressed the colony formation capacity of A549 and H1299 cells, while this influence was 
A

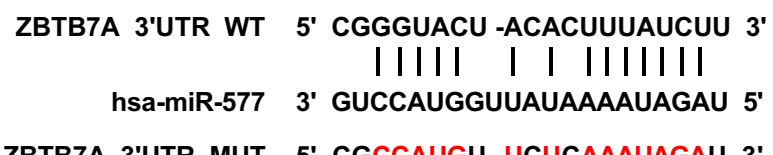

ZBTB7A 3'UTR MUT 5' CGCCAUGU -UCUCAAAUAGAU 3'
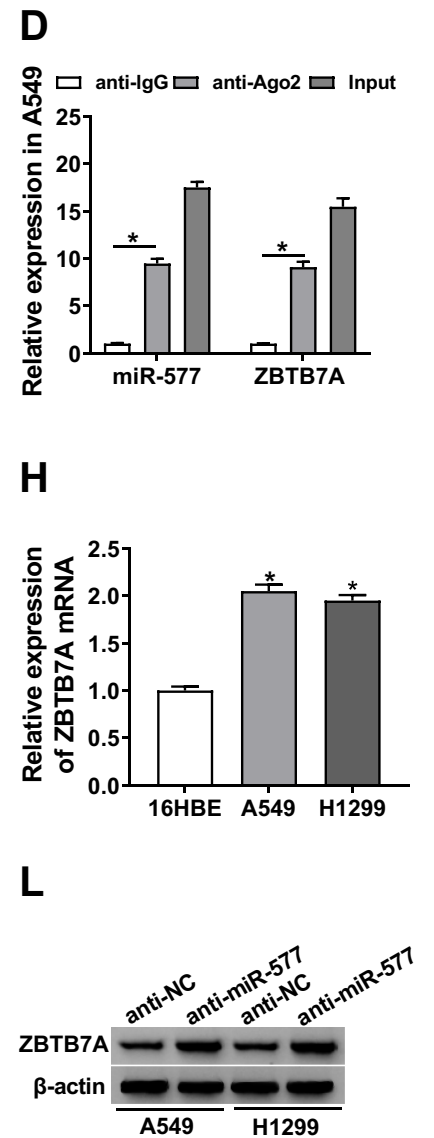

E
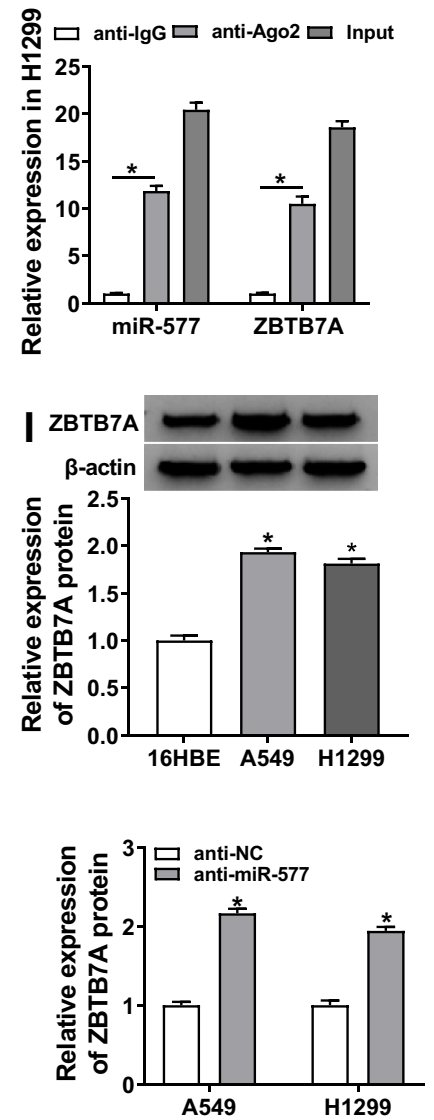

B

A549

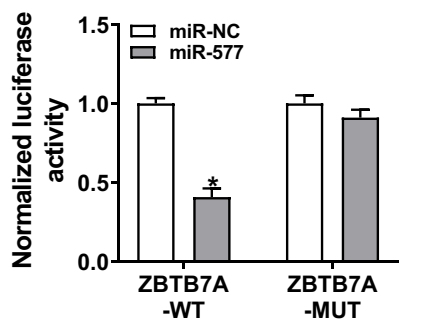

$\mathbf{F}$

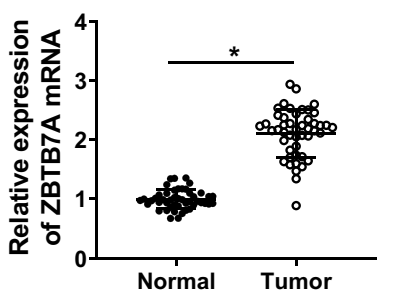

$\mathbf{J}$

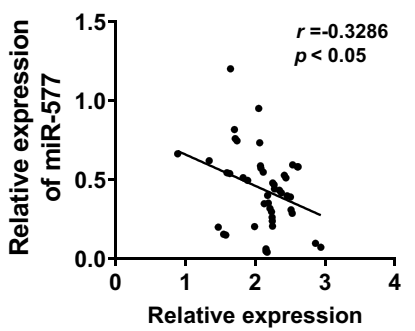

of ZBTB7A mRNA
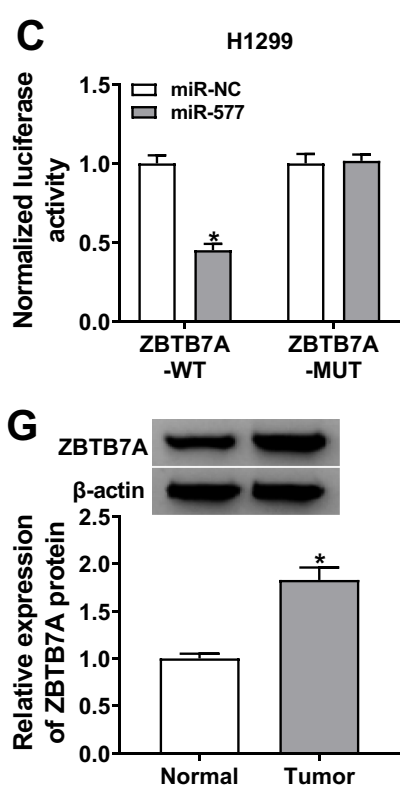

K

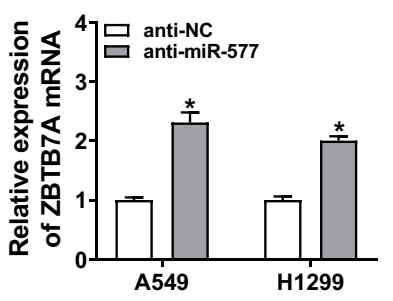

Figure 5 MiR-577 targeted ZBTB7A in NSCLC cells. (A) The binding sites between ZBTB7A and miR-577 were predicted with the starBase 3.0. (B and C) Dual-luciferase reporter assay was carried out to evaluate the luciferase activities of ZBTB7A-WT and ZBTB7A-MUT in A549 and HI299 cells transfected with miR-577 or miR-NC. (D and E) After RIP assay, the abundance of miR-577 and ZBTB7A was assessed via qRT-PCR. (F-I) The mRNA and protein levels of ZBTB7A in NSCLC tissues and neighboring normal tissues, as well as NSCLC cells (A549 and HI299 cells) and the 16HBE cells, were detected via qRT-PCR or Western blot analysis. (J) Pearson's correlation analysis was performed to analyze the correlation between ZBTB7A and miR-577 in NSCLC tissues. (K and L) QRT-PCR or Western blot analysis was executed for analysis the levels of ZBTB7A mRNA and protein in A549 and HI299 cells transfected with anti-miR-577 or anti-NC. $* P<0.05$.

abrogated by ZBTB7A overexpression (Figure 6B). Moreover, ZBTB7A enhancement reversed the suppressive effects of miR-577 upregulation on migration and invasion of A549 and H1299 cells (Figure 6C and D). Furthermore, ZBTB7A elevation overturned the reduction of ECAR of A549 and H1299 cells caused by miR-577 enhancement (Figure 6E). These data indicated that miR577 mediated cell colony formation, migration, invasion, and ECAR in NSCLC cells via ZBTB7A.

\section{Circ_0016760 Regulated ZBTB7A} Expression via miR-577 in NSCLC Cells

To probe into whether circ_0016760 regulated the expression of ZBTB7A in NSCLC cells via miR-577, we detected the protein level of ZBTB7A in NSCLC cells transfected with siNC, si-circ_0016760, circ_0016760+anti-NC, or circ_0016760+anti-miR-577. The data manifested that the protein level of ZBTB7A was repressed by circ_0016760 silencing in A549 and H1299 cells, while this influence 
A
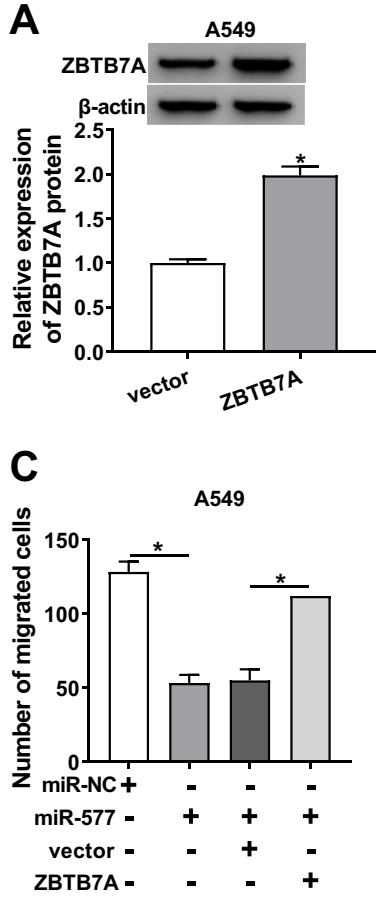

E

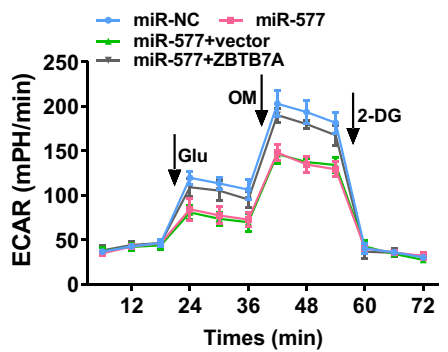

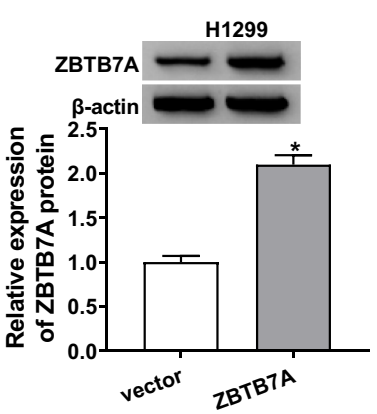
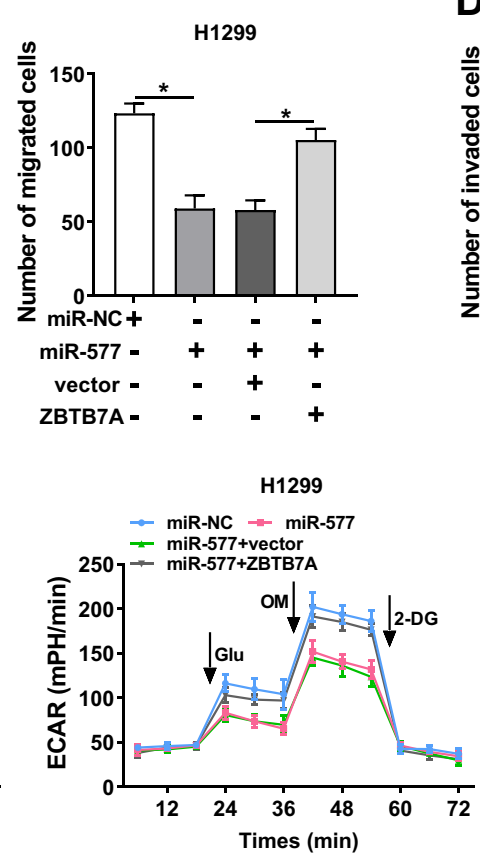
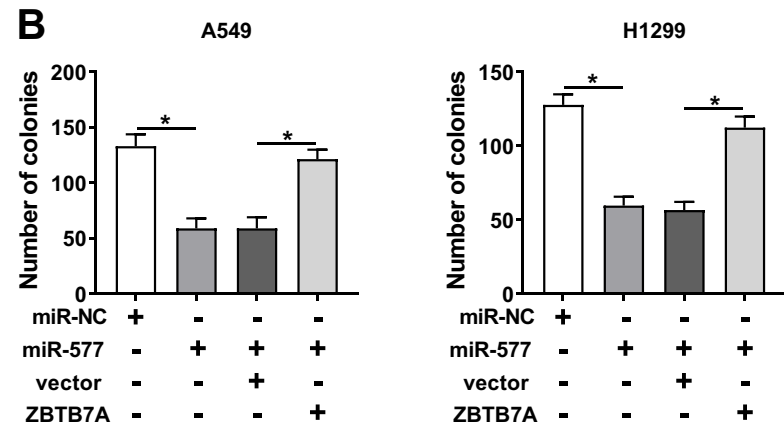

D
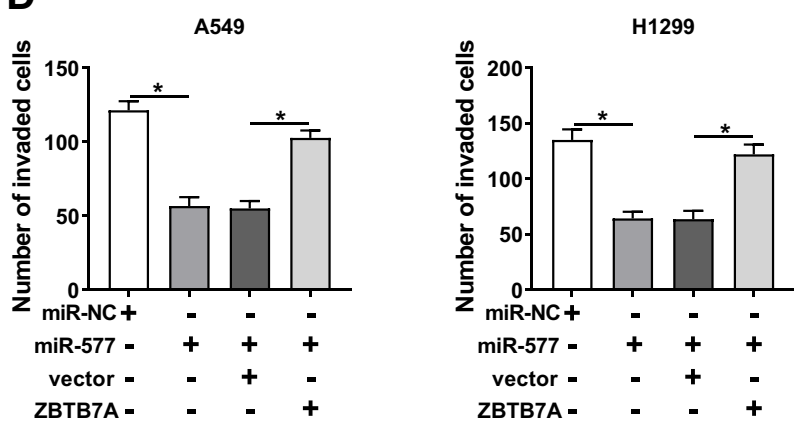

Figure 6 miR-577 played its roles through ZBTB7A in NSCLC cells. (A) Western blot analysis was executed for the evaluation of the protein level of ZBTB7A in A549 and HI299 cells transfected with ZBTB7A or vector. (B-E) A549 and HI299 cells were transfected with miR-NC, miR-577, miR-577+vector, or miR-577+ZBTB7A. (B) The colony formation activity of $A 549$ and $\mathrm{H} 1299$ cells was analyzed via colony formation assay. (C and D) Transwell assay was employed to determine the migration and invasion of A549 and HI299 cells. (E) ECAR assay was utilized to assess the ECAR of A549 and HI299 cells. *P $<0.05$.

was restored by miR-577 inhibition (Figure 7A and B). In sum, these data suggested that circ_0016760 regulated the expression of ZBTB7A via miR-577 in NSCLC cells.

\section{Circ_0016760 Silencing Repressed Tumor} Growth in vivo

Based on the above results, we established the mouse xenograft model to confirm the role of circ_0016760 in NSCLC. The results displayed that tumor volume and weight were effectively declined in the sh-circ_0016760 group when compared to the sh-NC group (Figure 8A and B). Also, circ_0016760 expression was reduced in the tumor tissues of the sh-circ_0016760 group relative to the sh-NC group, but miR-577 expression had a reverse trend (Figure 8C and D). And the level of ZBTB7A protein in tumor tissues of the sh-circ_0016760 group was decreased in comparison with the sh-NC group (Figure 8E). These results indicated that circ_0016760 downregulation curbed NSCLC growth in vivo.

\section{Discussion}

NSCLC is the main type of lung cancer, and its 5-year survival rate is less than $15 \%{ }^{29}$ Growing evidence disclosed that circRNAs were associated with the occurrence and development of diverse cancers. ${ }^{8-10}$ It was reported that circRNA was a key molecular modulator in NSCLC and could serve as a novel diagnostic biomarker and prognostic biomarker. ${ }^{30}$ Increasing evidence had disclosed 
A

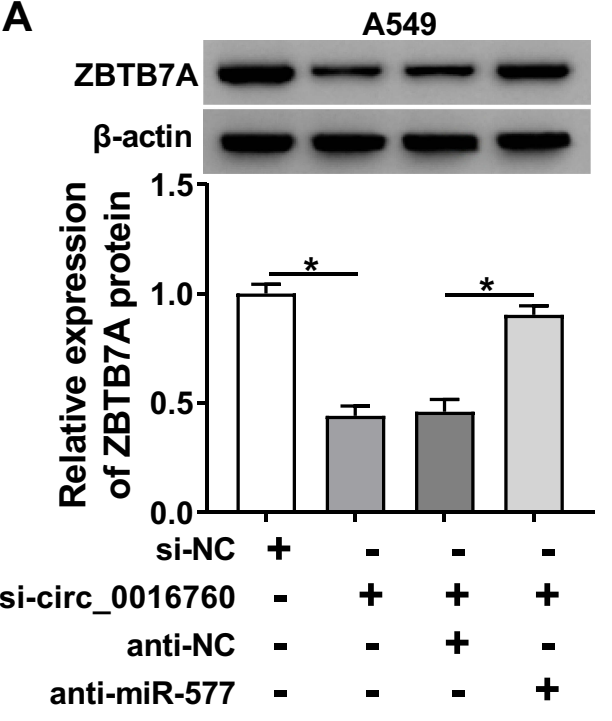

B

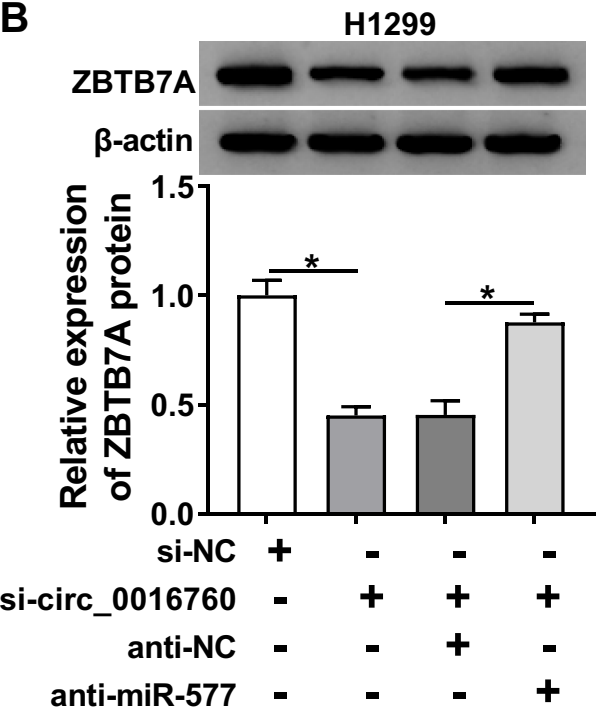

Figure 7 ZBTB7A was regulated by circ_0016760 through miR-577 in NSCLC cells. (A and B) Protein level of ZBTB7A in A549 and HI299 cells transfected with si-NC, sicirc_0016760, circ_0016760+anti-NC, or circ_0016760+anti-miR-577 was assessed through Western blot analysis. $* P<0.05$.

A

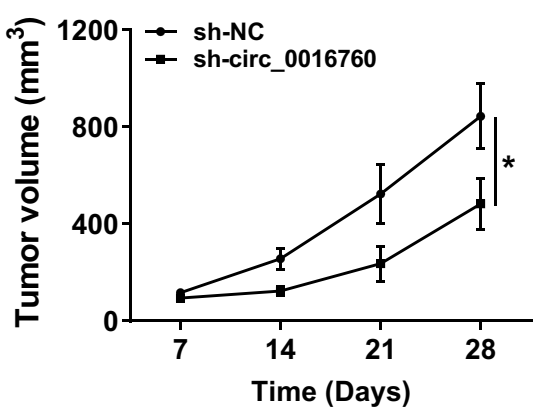

D

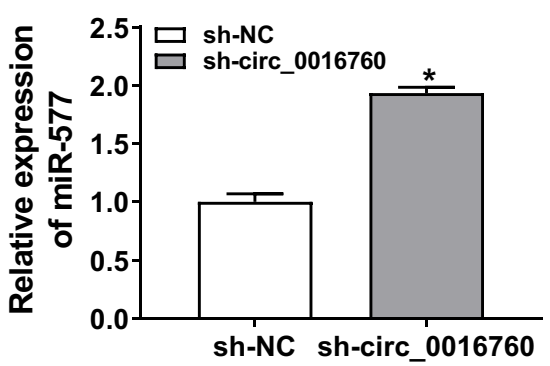

B

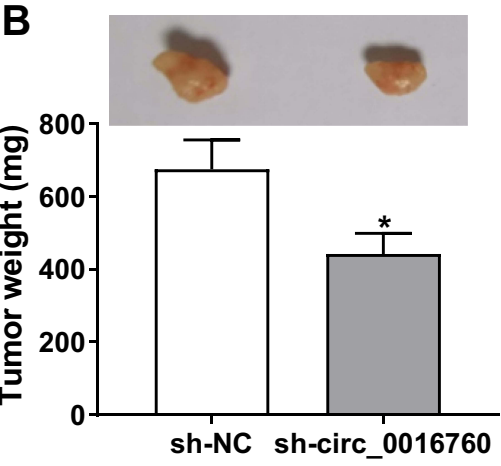

E

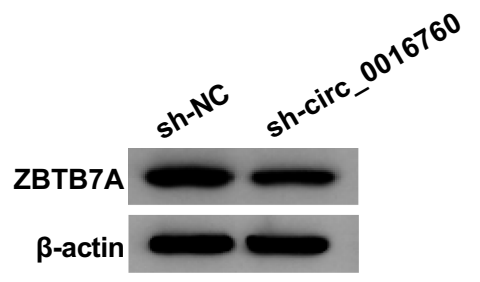

C
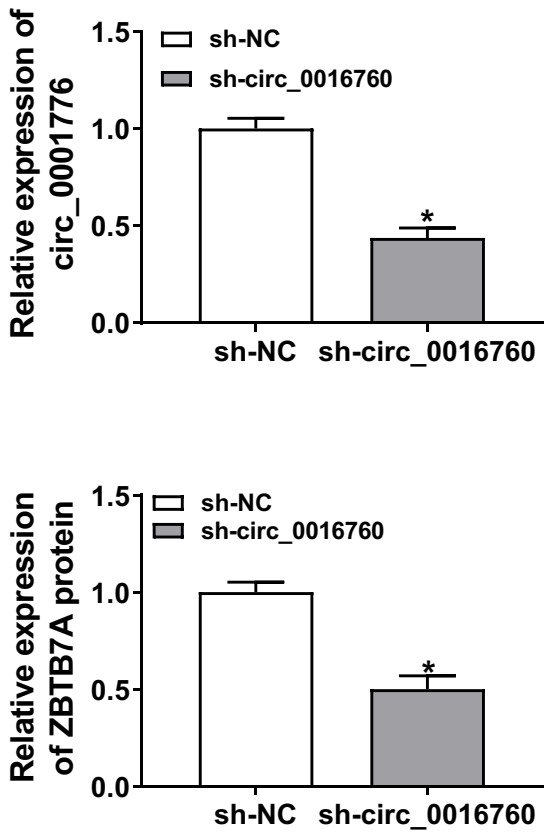

Figure 8 Effect of circ_0016760 exhaustion on tumor growth in vivo. (A) Tumor volume of the sh-circ_0016760 group and sh-NC group was measured every 7 days. (B) Tumor weight of the sh-circ_0016760 group and sh-NC group was assessed on day 28. (C and D) The expression of circ_0016760 and miR-577 in tumor tissues of the shcirc_0016760 and sh-NC groups was detected through qRT-PCR. (E) Protein level of ZBTB7A in tumor tissues of the sh-circ_00I6760 and sh-NC groups was evaluated via Western blot analysis. $* p<0.05$.

that circRNAs were related to the progression of NSCLC. Tan et al reported that circRNA circF-circEA-2a accelerated the invasion and migration of NSCLC cells. ${ }^{31}$ Moreover, circRNA circRNA_100146 impeded cell apoptosis and expedited cell invasion and proliferation in NSCLC cells through sponging miR-615-5p. ${ }^{32}$ Also, circRNA circPTPRA curbed NSCLC metastasis and epithelial-mesenchymal transition via sponging miR-96- 
$5 \mathrm{p} .{ }^{33}$ Herein, circ 0016760 was upregulated in NSCLC tissues and cells, and circ_0016760 silencing repressed colony formation, migration, invasion, and reduced ECAR of NSCLC cells. Report of $\mathrm{Li}$ et al pointed out that an apparent elevation of circ_0016760 was observed in NSCLC tissues and cells, and increased circ_0016760 expression repressed cell apoptosis and boosted cell metastatic properties and growth. ${ }^{12}$ Therefore, these findings revealed that circ 0016760 acted as an oncogene in NSCLC.

Increased researches revealed that circRNAs could act as a sponge for miRNAs to regulate tumor growth. ${ }^{8,10,33}$ For example, circRNA circLARP4 suppressed cell invasion and proliferation in gastric cancer cells through sponging miR-424-5p. ${ }^{8}$ In the present study, we discovered that miR-577 acted as a target for circ_0016760. MiR-577 was reported to be a suppressor in glioblastoma, papillary thyroid cancer, breast cancer, and colorectal cancer. ${ }^{16,17,34,35}$ The report of Zhang et al claimed that upregulated miR-577 expression impeded the growth of glioblastoma multiforme via blocking the Wnt signaling pathway. ${ }^{17}$ Also, the restoration of miR577 curbed cell colony formation, proliferation, and boosted cell cycle arrest in colorectal cancer cells. ${ }^{35}$ Moreover, miR-577 expression was reduced in breast cancer tissues, and miR-577 restoration hampered cell metastasis and epithelial-mesenchymal transition in breast cancer cells. ${ }^{34}$ And miR-577 overexpression repressed cell epithelial-mesenchymal transition and proliferation via WNT2B/Wnt/ $\beta$-catenin pathway in NSCLC cells. ${ }^{19}$ In the current study, miR-577 expression was declined in NSCLC tissues and cells, and miR-577 elevation repressed cell proliferation, migration, invasion, and reduced ECAR in NSCLC cells in vitro, which was in line with the above studies. ${ }^{19}$ Furthermore, miR-577 silencing overturned the inhibitory effects of circ_0016760 repression on colony formation, migration, invasion, and ECAR of NSCLC cells. However, miR577 expression was augmented in gastric cancer tissues and cells, and miR-577 enhancement facilitated gastric cancer chemoresistance and metastasis, which might be related to the tissue specificity. ${ }^{17}$ Therefore, we inferred that circ_0016760 modulated NSCLC progression via miR-577.

Additionally, we found that ZBTB7A served as a target for miR-577 in NSCLC cells. ZBTB7A had been reported to be acted as a context-dependent cancer gene that could play different functions in different cancers. Liu et al showed that inhibition of ZBTB7A expedited cell migration and invasion by enhancing MCAM expression in melanoma cells. ${ }^{22}$ Also, ZBTB7A impeded the SOX9-dependent pathway to impede prostate cancer progression. ${ }^{21}$ However, ZBTB7A could boost the chemoresistance of osteosarcoma by suppressing the activity linc_00473-IL24 by transcription. ${ }^{24}$ Mao et al also pointed out that increased ZBTB7A expression contributed to tumor metastasis and cell invasion and migration in breast cancer cells. ${ }^{23}$ Furthermore, miR-520e repressed the expression of ZBTB7A in NSCLC cells, which suppressed cancer cell invasion, proliferation, and migration. ${ }^{25}$ Herein, ZBTB7A expression was declined in NSCLC tissues and cells, and ZBTB7A overexpression reversed miR-577 inhibition-medicated impacts on colony formation, migration, invasion, and ECAR of NSCLC cells. Moreover, ZBTB7A was regulated by circ_0016760 through miR-577 in NSCLC cells. Hence, all data suggested that circ_0016760 knockdown repressed NSCLC progression via the miR-577/ZBTB7A axis.

In sum, circ_0016760 and ZBTB7A were upregulated and miR-577 was downregulated in NSCLC tissues and cells. Depletion of circ_0016760 impeded cell colony formation, migration, invasion, and reduced cell ECAR in NSCLC cells through the miR-577/ZBTB7A axis, providing a possible thought for NSCLC treatment.

\section{Highlights}

1. Circ_0016760 was upregulated in NSCLC tissues and cells.

2. Circ_0016760 depletion impeded the progression of NSCLC.

3. Circ_0016760 acted as a sponge for miR-577 in NSCLC cells.

4. ZBTB7A was regulated by circ_0016760 via sponging miR-577 in NSCLC cells.

\section{Data Sharing Statement}

The analyzed data sets generated during the present study are available from the corresponding author on reasonable request.

\section{Ethics Approval and Consent to Participate}

The present study was approved by the ethical review committee of the First Hospital of Yulin. 


\section{Acknowledgment}

Yanhong Hao and Junfeng Xi are co-first authors for this study.

\section{Funding}

This work was supported by the Natural Science Basic Research Program of Shaanxi (Program No. 2018JM7068).

\section{Disclosure}

The authors declare that they have no competing interests.

\section{References}

1. Aggarwal A, Lewison G, Idir S, et al. The state of lung cancer research: a global analysis. $J$ Thorac Oncol. 2016;11(7):1040-1050. doi:10.1016/j.jtho.2016.03.010

2. Bray F, Ferlay J, Soerjomataram I, Siegel RL, Torre LA, Jemal A. Global cancer statistics 2018: GLOBOCAN estimates of incidence and mortality worldwide for 36 cancers in 185 countries. CA Cancer J Clin. 2018;68(6):394-424. doi:10.3322/caac.21492

3. Zheng M. Classification and pathology of lung cancer. Surg Oncol Clin N Am. 2016;25(3):447-468. doi:10.1016/j.soc.2016.02.003

4. Herbst RS, Morgensztern D, Boshoff C. The biology and management of non-small cell lung cancer. Nature. 2018;553 (7689):446-454. doi:10.1038/nature25183

5. Pamudurti NR, Bartok O, Jens M, et al. Translation of CircRNAs. Mol Cell. 2017;66(1):9-21.e27. doi:10.1016/j.molcel.2017.02.021

6. Chen LL. The biogenesis and emerging roles of circular RNAs. Nat Rev Mol Cell Biol. 2016;17(4):205-211. doi:10.1038/nrm.2015.32

7. Huang MS, Zhu T, Li L, et al. LncRNAs and CircRNAs from the same gene: masterpieces of RNA splicing. Cancer Lett. 2018;415:49-57. doi:10.1016/j.canlet.2017.11.034

8. Zhang J, Liu H, Hou L, et al. Circular RNA_LARP4 inhibits cell proliferation and invasion of gastric cancer by sponging miR-424-5p and regulating LATS1 expression. Mol Cancer. 2017;16(1):151.

9. Zhong Z, Huang M, Lv M, et al. Circular RNA MYLK as a competing endogenous RNA promotes bladder cancer progression through modulating VEGFA/VEGFR2 signaling pathway. Cancer Lett. 2017;403:305-317. doi:10.1016/j.canlet.2017.06.027

10. Yang R, Xing L, Zheng X, Sun Y, Wang X, Chen J. The circRNA circAGFG1 acts as a sponge of miR-195-5p to promote triple-negative breast cancer progression through regulating CCNE1 expression. Mol Cancer. 2019;18(1):4.

11. Bi W, Huang J, Nie C, et al. CircRNA circRNA_102171 promotes papillary thyroid cancer progression through modulating CTNNBIP1-dependent activation of beta-catenin pathway. $J$ Exp Clin Cancer Res. 2018;37(1):275. doi:10.1186/s13046-018-0936-7

12. Li Y, Hu J, Li L, et al. Upregulated circular RNA circ_0016760 indicates unfavorable prognosis in NSCLC and promotes cell progression through miR-1287/GAGE1 axis. Biochem Biophys Res Commun. 2018;503(3):2089-2094. doi:10.1016/j.bbrc.2018.07.164

13. Hayes J, Peruzzi PP, Lawler S. MicroRNAs in cancer: biomarkers, functions and therapy. Trends Mol Med. 2014;20(8):460-469. doi:10.1016/j.molmed.2014.06.005

14. Kwak PB, Iwasaki S, Tomari Y. The microRNA pathway and cancer. Cancer Sci. 2010;101(11):2309-2315. doi:10.1111/j.13497006.2010.01683.x

15. Luo Y, Wu J, Wu Q, et al. miR-577 regulates TGF-beta induced cancer progression through a SDPR-modulated positive-feedback loop with ERK-NF-kappaB in gastric cancer. Mol Ther. 2019;27 (6):1166-1182. doi:10.1016/j.ymthe.2019.02.002
16. Xue KC, Hu DD, Zhao L, Li N, Shen HY. MiR-577 inhibits papillary thyroid carcinoma cell proliferation, migration and invasion by targeting SphK2. Eur Rev Med Pharmacol Sci. 2017;21(17):3794-3800.

17. Zhang W, Shen C, Li C, et al. miR-577 inhibits glioblastoma tumor growth via the Wnt signaling pathway. Mol Carcinog. 2016;55 (5):575-585. doi:10.1002/mc.22304

18. Wang $\mathrm{Y}, \mathrm{Lu} \mathrm{Z}$, Wang $\mathrm{N}$, et al. Long noncoding RNA DANCR promotes colorectal cancer proliferation and metastasis via miR-577 sponging. Exp Mol Med. 2018;50(5):57.

19. Wang B, Sun L, Li J, Jiang R. miR-577 suppresses cell proliferation and epithelial-mesenchymal transition by regulating the WNT2B mediated Wnt/beta-catenin pathway in non-small cell lung cancer. Mol Med Rep. 2018;18(3):2753-2761. doi:10.3892/ mmr.2018.9279

20. Men L, Nie D, Nie H. microRNA577 inhibits cell proliferation and invasion in nonsmall cell lung cancer by directly targeting homeobox A1. Mol Med Rep. 2019;19(3):1875-1882. doi:10.3892/ mmr.2019.9804

21. Wang G, Lunardi A, Zhang J, et al. Zbtb7a suppresses prostate cancer through repression of a Sox9-dependent pathway for cellular senescence bypass and tumor invasion. Nat Genet. 2013;45(7):739-746. doi:10.1038/ng.2654

22. Liu XS, Genet MD, Haines JE, et al. ZBTB7A suppresses melanoma metastasis by transcriptionally repressing MCAM. Mol Cancer Res. 2015;13(8):1206-1217. doi:10.1158/1541-7786.MCR-15-0169

23. Mao A, Chen M, Qin Q, et al. ZBTB7A promotes migration, invasion and metastasis of human breast cancer cells through NF-kappaBinduced epithelial-mesenchymal transition in vitro and in vivo. J Biochem. 2019;166(6):485-493. doi:10.1093/jb/mvz062

24. Zhang L, Wang Y, Li X, et al. ZBTB7A enhances osteosarcoma chemoresistance by transcriptionally repressing IncRNALINC00473-IL24 activity. Neoplasia. 2017;19(11):908-918. doi:10.1016/j.neo.2017.08.008

25. Zhijun Z, Jingkang H. MicroRNA-520e suppresses non-small-cell lung cancer cell growth by targeting Zbtb7a-mediated Wnt signaling pathway. Biochem Biophys Res Commun. 2017;486(1):49-56. doi:10.1016/j.bbrc.2017.02.121

26. Danan M, Schwartz S, Edelheit S, Sorek R. Transcriptome-wide discovery of circular RNAs in Archaea. Nucleic Acids Res. 2012;40 (7):3131-3142. doi:10.1093/nar/gkr1009

27. Li W, Ma H, Sun J. MicroRNA34a/c function as tumor suppressors in Hep2 laryngeal carcinoma cells and may reduce GALNT7 expression. Mol Med Rep. 2014;9(4):1293-1298. doi:10.3892/ mmr.2014.1929

28. Sun K, Tang S, Hou Y, et al. Oxidized ATM-mediated glycolysis enhancement in breast cancer-associated fibroblasts contributes to tumor invasion through lactate as metabolic coupling. EBioMedicine. 2019;41:370-383. doi:10.1016/j.ebiom.2019.02.025

29. Ge L, Shi R. Progress of EGFR-TKI and ALK/ROS1 inhibitors in advanced non-small cell lung cancer. Int J Clin Exp Med. 2015;8 (7):10330-10339.

30. Li C, Zhang L, Meng G, Wang Q, Lv X, Zhang J. Circular RNAs: pivotal molecular regulators and novel diagnostic and prognostic biomarkers in non-small cell lung cancer. J Cancer Res Clin Oncol. 2019;145(12):2875-2889. doi:10.1007/s00432-019-03045-4

31. Tan S, Sun D, Pu W, et al. Circular RNA F-circEA-2a derived from EML4-ALK fusion gene promotes cell migration and invasion in non-small cell lung cancer. Mol Cancer. 2018;17(1):138.

32. Chen L, Nan A, Zhang N, et al. Circular RNA 100146 functions as an oncogene through direct binding to miR-361-3p and miR-615-5p in non-small cell lung cancer. Mol Cancer. 2019;18(1):13. doi:10.1186/ s12943-019-0943-0

33. Wei S, Zheng Y, Jiang Y, et al. The circRNA circPTPRA suppresses epithelial-mesenchymal transitioning and metastasis of NSCLC cells by sponging miR-96-5p. EBioMedicine. 2019;44:182-193. doi:10.1016/j.ebiom.2019.05.032 
34. Yin C, Mou Q, Pan X, Zhang G, Li H, Sun Y. MiR-577 suppresses epithelial-mesenchymal transition and metastasis of breast cancer by targeting Rab25. Thorac Cancer. 2018;9(4):472-479.
35. Jiang H, Ju H, Zhang L, Lu H, Jie K. microRNA-577 suppresses tumor growth and enhances chemosensitivity in colorectal cancer. J Biochem Mol Toxicol. 2017;31(6):e21888.

\section{Publish your work in this journal}

Cancer Management and Research is an international, peer-reviewed open access journal focusing on cancer research and the optimal use of preventative and integrated treatment interventions to achieve improved outcomes, enhanced survival and quality of life for the cancer patient.
The manuscript management system is completely online and includes a very quick and fair peer-review system, which is all easy to use. Visit http://www.dovepress.com/testimonials.php to read real quotes from published authors. 